\title{
Ruptured neoatherosclerosis as source of late stent thrombosis
}

\author{
Mathieu Stadelmann, Jérémy Valentin, Marie-Noelle Giraud, Stéphane Cook \\ Department of Cardiology, University and Hospital, Fribourg, Switzerland
}

\section{Summary}

Neoatherosclerosis has been termed to define neointimal atherosclerotic changes occurring within a coronary stent [1, 2]. Based on histological [2] and angioscopic studies [3], rupture within the neointima has been reported in the long-term after coronary stent im- plantation and has been proposed to be responsible for late and very late stent thrombosis. More recently, optical coherence tomography (OCT) with high discriminating power has been proposed to characterise this phenomenon in daily practice $[4,5]$.

Key words: stent thrombosis; neoatherogenesis; intravascular; imaging

Figure 1

Representative coronary angiograms before (panel A), during (panel B) and after percutaneous coronary intervention (panel C). Panels D-J are representative optical coherence tomography (OCT) findings; panel D depicts the stent area seen in the longitudinal view; panels E-J: cross-sectional images demonstrating neoatherogenesis with abundant microvessels (MV), ruptured membrane and cauliflower-like thrombosis (T). Panels K-M show illustrative histological findings of the thrombus harvested from the target-vessel. Panels K-L: Movat pentachrom staining underlines the nicely delineated neointimal hyperplasia attached to the thrombus (T). Panel M: HRP Anti Macrophage [MAC387] staining permits to delineate macrophages in the loose part of the plaque.
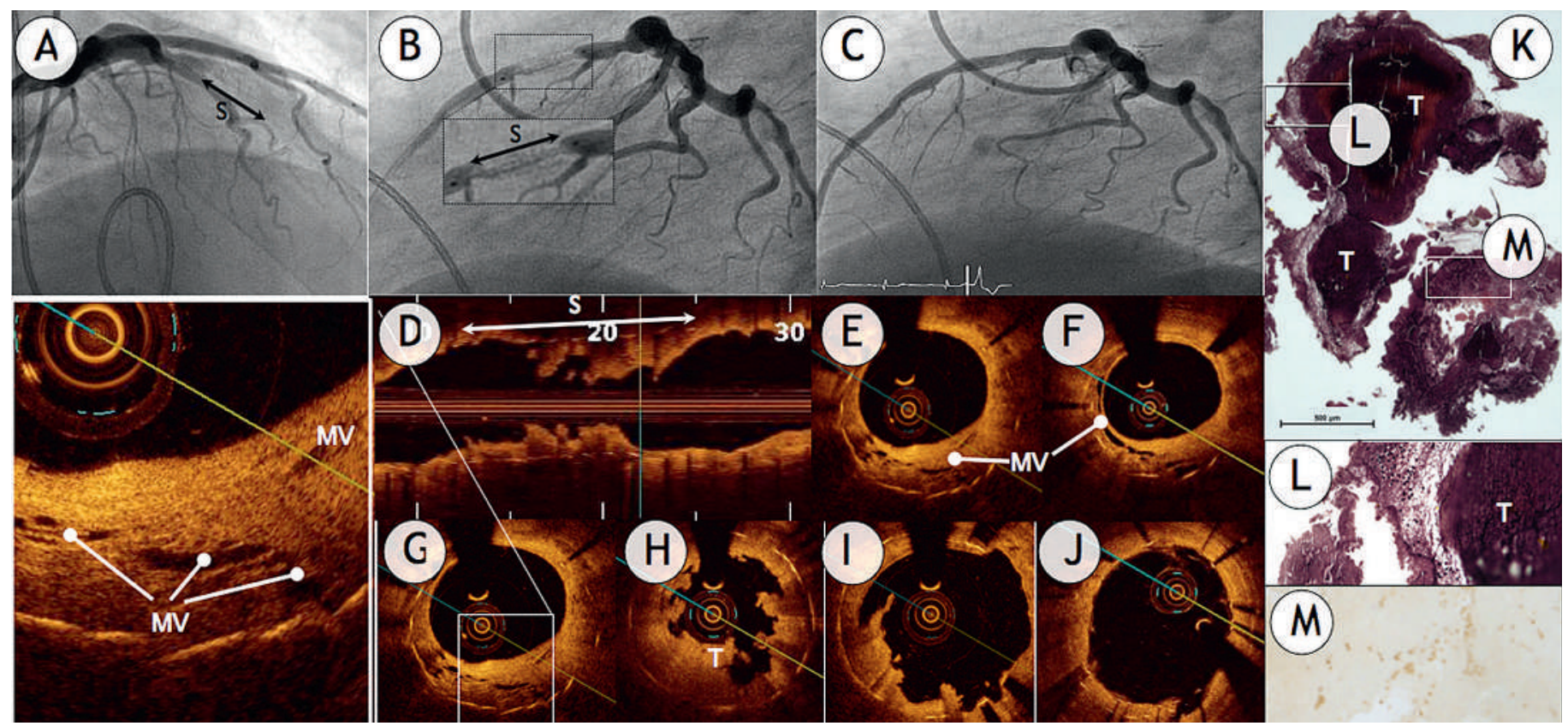

\footnotetext{
Funding / potential competing interests:

FSC Fonds Scientifique Cardiovasculaire, Fribourg, Switzerland. No other potential conflict of interest relevant to this article was reported.
}

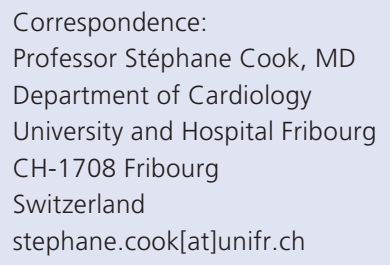




\section{Case report}

We report the case of a 72 -year-old man presenting with anterior ST-elevation myocardial infarction 2,833 days after bare-metal stent implantation (Multi-link Vision, Abbott Vasc, $3.5 \times 28 \mathrm{~mm}$ ) in the mid portion of the left anterior descending coronary artery. The coronary angiogram (fig. 1A) demonstrated an occlusion at stent (S) entrance. Percutaneous coronary intervention was readily performed and the aspiration thrombectomy permitted a restoration to a normal coronary blood flow (fig. 1B). Subsequently, an OCT imaging (DragonFly catheter, LightLab Imaging, St. Jude Medical) demonstrated "spongiform" neoatherosclerosis due to redundant intraintimal neovascularisation (fig. 1D-G) with ruptured thin-cap fibroatheroma, and reactive "cauliflower" thrombosis (fig. $1 \mathrm{H}-\mathrm{I}$ ). Histology of the aspirate demonstrated area of nicely delineated neointimal hyperplasia attached to the thrombus (fig. 1K-L, Movat pentachrom staining). Some sparse foamy macrophages are present within the restenotic area, close to the thrombus area and in larger number in the weak part of the plaque (fig. 1M, HRP Anti Macrophage [MAC387] staining). The lesion was then treated with balloon angioplasty with nice angiographic end-result (fig. 1C).

Stent thrombosis is a rare but devastating event. Very late stent thrombosis ( $>1$ year after stent implantation) is a specific entity, mainly encountered after implantation of first-generation drug eluting stents with an observed rate at $0.6 \%$ events/year [6]. This rare condition is usually due to hypersensitivity reaction as- sociated with delayed endothelial healing, exaggerated positive vessel remodeling and incomplete stent apposition [7]. Nevertheless, some cases might be due to the rupture of the neointimal tissue. Indeed, and although in-stent restenosis is generally considered as a stable process, growing evidence - as illustrated here - tends to challenge this concept. In this case and in analogy to de novo atherosclerotic plaque, the presence of abundant microvessels seems to be a key player in the neointimal plaque vulnerability.

\section{References}

1 Chen MS, John JM, Chew DP, Lee DS, Ellis SG, Bhatt DL. Bare metal stent restenosis is not a benign clinical entity. Am Heart J. 2006;151: 1260-4.

2 Nakazawa G, Otsuka F, Nakano M, Vorpahl M, Yazdani SK, Ladich E, et al. The pathology of neoatherosclerosis in human coronary implants bare-metal and drug-eluting stents. J Am Coll Cardiol. 2011;57: $1314-22$.

3 Higo T, Ueda Y, Oyabu J, Okada K, Nishio M, Hirata A, et al. Atherosclerotic and thrombogenic neointima formed over sirolimus drug-eluting stent: An angioscopic study. JACC Cardiovasc Imaging. 2009;2: 616-24.

4 Kang SJ, Mintz GS, Akasaka T, Park DW, Lee JY, Kim WJ, et al. Optical coherence tomographic analysis of in-stent neoatherosclerosis after drug-eluting stent implantation. Circulation. 2011;123:2954-63.

5 Takano M, Yamamoto M, Inami S, Murakami D, Ohba T, Seino Y, et al. Appearance of lipid-laden intima and neovascularization after implantation of bare-metal stents extended late-phase observation by intracoronary optical coherence tomography. J Am Coll Cardiol. 2009;55: 26-32.

6 Wenaweser P, Daemen J, Zwahlen M, van Domburg R, Juni P, Vaina S, et al. Incidence and correlates of drug-eluting stent thrombosis in routine clinical practice: 4-year results from a large 2 -institutional cohort study. J Am Coll Cardiol. 2008;52:1134-40.

7 Cook S, Wenaweser P. Off-label use and the spectre of drug-eluting stent thrombosis. Circulation. Cardiovascular interventions. 2009;2:273-6. 